\section{rev Psi}

Revista de Psicología (UNLP)

https://revistas.unlp.edu.ar/revpsi

Artículo de investigación

\title{
Perfiles epidemiológicos de personas con discapacidad beneficiarias de políticas públicas a través del WHO-DAS II
}

María José Bagnato ${ }^{1}$

Correspondencia

majose@psico.edu.uy

Filiaciones institucionales

${ }^{1}$ Facultad de Psicología, Universidad de la República (Uruguay)
Julia Córdoba ${ }^{1}$

\section{Resumen}

El presente estudio pretende mostrar la utilidad del World Health Organization Disability Assessment Schedule 2.0 (WHO-DAS II) (OMS, 2009) para analizar el perfil epidemiológico de personas con discapacidad. Se aplica, a su vez, un cuestionario que recoge información relacionada con la salud y la discapacidad de 247 usuarios/ as de prestaciones públicas de la ciudad de Montevideo (Uruguay). Se proponen como objetivos identificar el perfil poblacional con discapacidad entrevistado, relacionar el uso de la prestación que declaran con los espacios sociosanitarios en los que participa, determinar las necesidades de apoyo para la realización de las actividades de la vida diaria e identificar necesidades de acceso a servicios e inclusión social. Se concluye que es posible utilizar el WHO-DASII (OMS, 2009) para conocer el perfil poblacional y, en detalle, la existencia de barrearas y facilitadores para la participación en comunidad, ocio, acceso a la salud e inclusión laboral y educativa.

\section{Palabras clave}

discapacidad | CIF | políticas públicas | salud

\section{Cómo citar}

Bagnato, M. J. y Córdoba, J. (2021).

Perfiles epidemiológicos de personas con discapacidad beneficiarias de políticas públicas a través del WHO-DAS II. Revista de Psicología, 20(2), 18-33. HTTP://DX.DOI. ORG/10.24215/2422572XE075
Recibido

18 jun. 2020

Aceptado

24 nov. 2020

Publicado

28 nov. 2020
ISSN

2422-572X

Licencia

(c) Copyright: Bagnato, M. J. y

Córdoba, J. Licencia de Cultura Libre CC-BY 4.0

Entidad editora

RevPsi es una publicación de la

Facultad de Psicología (Universidad

Nacional de La Plata, Argentina) 


\section{Perfis epidemiológicos de pessoas com deficiência beneficiárias de políticas públicas por meio do WHO-DAS II}

\section{Resumo}

Este estudo tem como objetivo mostrar a utilidade do Cronograma de Avaliação de Deficiência da Organização Mundial da Saúde 2.0 (OMS-DAS II) (OMS, 2009) para analisar o perfil epidemiológico de pessoas com deficiência. Por sua vez, é aplicado um questionário que coleta informações relacionadas à saúde e invalidez de 247 usuários de benefícios públicos na cidade de Montevidéu (Uruguai). Os objetivos são identificar o perfil da população entrevistada com deficiência, relacionar o uso do benefício que declaram com os espaços sócio-sanitários em que participam, determinar as necessidades de apoio para a realização de atividades da vida diária e identificar necessidades de acesso a serviços e inclusão social. Conclui-se que é possível utilizar a OMS-DASII (OMS, 2009) para conhecer o perfil da população e, em detalhes, a existência de barreiras e facilitadores para a participação na comunidade, lazer, acesso à saúde e inclusão trabalhista e educacional.

\section{Palavras-chave}

deficiência $\mid$ CIF | políticas públicas | saúde

\section{Epidemiological profiles of people with disabilities beneficia- ries of public policies through the WHO-DAS II}

\section{Abstract}

This study aims to show the usefulness of the World Health Organization Disability Assessment Schedule 2.0 (WHO-DAS II) (WHO, 2009) to analyze the epidemiological profile of people with disabilities. We administered a questionnaire that collected information related to the health and disability of 247 public services users in Montevideo (Uruguay). The objectives were to identify the profile of the disabled interviewees, to relate their use of public services with the socio-sanitary spaces in which they participate, to determine the support needed for carrying out daily activities, and to identify their needs for access to services and social inclusion. It is concluded that it is possible to use the WHO-DASII (WHO, 2009) to get a profile of the population and, more specifically, to explore the existence of barriers and facilitators for participation in the community and inclusion regarding leisure, health, labor, and education.

\section{Keywords}

disability | ICF | public policy | health 


\section{Aspectos destacados del trabajo}

- El WHODAS II (OMS, 2010) permite evaluar y caracterizar la discapacidad.

- Las personas entrevistadas utilizan las prestaciones principalmente para actividades vinculadas a su condición de salud.

- Las personas entrevistadas en su mayoría desconocen el origen de su discapacidad.

- La información recabada deja en claro la escasa inclusión y participación social de los entrevistados.

El "Informe mundial sobre discapacidad" (OMS, 2011), plantea como insuficiente el acceso de las personas en situación de discapacidad a los sistemas de salud (para prevención, control, tratamiento y rehabilitación), así como también la oferta de los mismos. Las personas con discapacidad tienen mayor riesgo a contraer problemas de salud que las personas sin discapacidades (Jiménez Lara, 2010) y aun así no tienen un acceso equitativo a las prestaciones de salud (Tamayo, Besoaín y Rebolledo, 2018) lo que genera, en esta población, una alta tasa de comorbilidad. Esto sucede en el contexto de una proyección en aumento de las personas con discapacidad debido al envejecimiento de la población, una mayor esperanza de vida y la incidencia de las enfermedades crónicas.

Algunos países de América Latina han abordado la temática de la discapacidad desde una mirada epidemiológica, por ejemplo, Chile (Instituto Nacional de Estadísticas [INE], 2004), Ecuador (Ortiz Segarra, 2013), Cuba (Cobas Ruíz et al., 2010).

Tal como plantean Ballerini, Figueras y Del Carlo (2020) en Latinoamérica se han dado diferentes luchas para lograr sistemas de salud más equitativos y de cobertura universal, sin embargo, no se ha logrado en su totalidad en la mayoría de los países de corte neoliberal.

Aún en aquellos con mayores avances la discapacidad no suele considerarse dentro de dicha universalidad como población que requiere de los componentes de accesibilidad física, comunicacional, administrativa y tecnológica.

Uruguay aún no cuenta con estudios epidemiológicos nacionales dirigidos a identificar los procesos de salud-enfermedad que viven las personas con discapacidad. La información oficial sobre este colectivo de personas en Uruguay surge del Censo de Población y Vivienda 2011 (Núñez, 2011) donde se incorpora por primera vez un módulo de discapacidad, sobre la base de las preguntas del Grupo de Washington, sin embargo, no recoge información acerca de acceso a la salud. Ello implica una falta de información sobre la discapacidad en Uruguay, así como del funcionamiento de los programas, servicios y prestaciones, lo que dificulta la evaluación, el rediseño e implementación de los mismos. 
Desde un enfoque integral, pensar la salud de las personas con discapacidad implica pensar una estrategia de "escenarios de la vida" (Jiménez Lara, 2010, p. 72) y obliga a considerar cómo los Determinantes Sociales de la Salud (DSS) -políticas, planes y servicios- impactan en la vida cotidiana de estas personas.

La epidemiología aplicada a la discapacidad se basa en la identificación de barreras y de facilitadores que compensan o contribuyen a la aparición de la discapacidad y/o la dependencia (Alarcón, 2009). Hay cuatro grandes productos que surgen de la relación entre epidemiología y discapacidad: la formulación de políticas sanitarias, la planificación de acciones a nivel nacional e internacional, la evaluación de servicios y programas ya existentes y la investigación científica (Rodriguez Da Silva, 2006). La posible retroalimentación de estos productos repercute directamente en la calidad de vida de las personas con discapacidad y sus familiares o referentes.

Enfocar la discapacidad desde esta perspectiva obliga a entenderla como un determinante social de la salud (Tamayo, Besoaín y Rebolledo, 2018) ya que estos se caracterizan por ser los aspectos contextuales en que una persona se desarrolla (nace, crece, vive, trabaja y envejece) y que condicionan los hábitos y la toma de decisiones vinculadas a la salud. Están sujetos a la economía, al poder y a los recursos existentes a nivel local, nacional y mundial, absolutamente influenciados por las políticas implementadas. Cada factor tiene incidencia por sí mismo y a la vez se interrelacionan, son causales de riesgo o protectores (Tamayo, Besoaín y Rebolledo, 2018). La discapacidad es considerada un DSS en tanto es generada por situaciones que restringen la participación, generan exclusión y aumentan el riesgo de pobreza.

El presente estudio, se desarrolla en unainstitución pública, la Intendencia de Montevideo (IM), a partir de las prestaciones dirigidas a personas con discapacidad. Asimismo, se enmarca en una actividad de formación curricular de práctica preprofesional de la Facultad de Psicología de la Universidad de la República de Uruguay.

El estudio se construye sobre la base de la prestación denominada Tarjeta de Gestión Social (TGS) para personas que presenten discapacidad y que también se otorga transitoriamente a quienes se encuentren realizando tratamiento por enfermedad. La TGS ofrece beneficios para el acceso gratuito al transporte capitalino y a los espectáculos culturales y deportivos. Según los datos aportados por la Intendencia de Montevideo, anualmente se realizan un promedio de 12.000 gestiones vinculadas a la TGS, ya sea por primera vez o por renovación.

El criterio de otorgamiento de la prestación es mediante la constatación de certificados médicos que den cuenta de los diagnósticos médicos asociados a discapacidad y/o tratamientos por enfermedad que tenga la persona interesada en la gestión. Tiene, a su vez, una adjudicación directa a aquellas personas que perciban pensión por invalidez del Banco de Previsión Social (BPS). La información sobre la que se aplican los criterios de otorgamiento de la prestación, por tanto, son brindados por otros actores/instituciones estatales los cuales tienen, cada uno de ellos, distintas formas de valorar las limitaciones de las personas y su situación de discapacidad. Aplicar el WHO DAS II (OMS, 2009) en este contexto, nos permite homologar los 
criterios para determinar el grado de discapacidad de las personas participantes del estudio. Uno de los beneficios del instrumentos es la valoración de la persona con determinada condición de salud en relación a su contexto, ya que las personas aunque puedan tener el mismo diagnóstico, sus experiencias vitales son distintas, así como las oportunidades para contar con apoyos, donde también se deben tener en cuenta los recursos personales (Ayuso Mateos, Nieto-Moreno, Sánchez-Moreno y Vázquez-Barquero, 2006).

Si bien se parte de la información técnica que surge de los diagnósticos, estos no dan información sobre cuáles son las funciones y las actividades que esta condición de salud ha afectado y cuáles no; del mismo modo, la información generada de esta manera es insuficiente a la hora de explicar cómo hacen uso de los servicios los beneficiarios, en este caso de la TGS. Como plantean Tamayo, Besoaín y Rebolledo (2018), al considerar la situación de discapacidad como sinónimo de estado de salud en lugar de entenderla como el contexto de circunstancias sociales, de salud y los factores ambientales que interaccionan negativamente entre sí (OMS, 2001), las intervenciones que se diseñen no tendrán el impacto que se desea ya que faltarán abordar aspectos fundamentales para la mejora de la calidad de vida de estas personas y la de su familia.

Uruguay cuenta con la Ley 18.651 de Protección Integral a las Personas con Discapacidad (LPIPD, 2010) en la que se establece la creación de un órgano, encargado de realizar la certificación única de discapacidad; para esto deben establecerse tanto los mecanismos, como los criterios de valoración válidos para todo el país. Dentro de las recomendaciones para la implementación de este órgano, está la adopción de instrumentos basados en la CIF (OMS, 2001). Desde la academia, se considera relevante aportar estudios, que permitan dar insumos para la adopción de dichos instrumentos y criterios, esta investigación pretende contribuir en tal sentido. La CIF (OMS, 2001) pone atención en el funcionamiento y la discapacidad de las personas en relación con su estado de salud, transformando en información complementaria las clasificaciones vinculadas con los diagnósticos. Por otro lado, como recoge la perspectiva del paciente, junto con el análisis funcional de la persona, se vuelve posible el abordaje desde un modelo biopsicosocial desde el punto de vista clínico y científico (Ayuso Mateos, Nieto-Moreno, Sánchez-Moreno y Vázquez-Barquero, 2006).

El objetivo general de este trabajo es analizar la utilidad del WHO-DAS II (OMS, 2009) para generar un perfil epidemiológico de la población con discapacidad que gestiona la prestación de políticas públicas. Para esto se plantean los siguientes objetivos específicos: a. identificar, según el grado de discapacidad, cuál es el perfil de la población con discapacidad que accede a la gestión de la prestación de la TGS de la IM; b. relacionar el uso del beneficio que declaran los entrevistados con los espacios sociosanitarios en los que ya participa; c. determinar las necesidades de apoyo por parte de los entrevistados para la realización de las actividades de la vida diaria; d. identificar las necesidades de acceso e inclusión de las personas que gestionan la tarjeta de gestión social para la discapacidad. 


\section{Método}

\section{Diseño}

Se trata de un estudio de corte cuantitativo, retrospectivo, que analiza los resultados de 247 participantes entrevistados, que inician o renuevan el trámite de solicitud para obtener la TGS entre abril de 2017 y setiembre de 2018 en la IM. Esta prestación es otorgada a quienes cumplan con al menos uno de los criterios de estar realizando rehabilitación, cuenten con Pensión por invalidez (BPS) y/o presentan determinados diagnósticos, que, luego de una entrevista médica, se les adjudica, además de la prestación, el tiempo de vigencia de esta.

\section{Participantes}

Se presentan los resultados obtenidos de un total de 247 personas entrevistadas. Este número es muy inferior al número de solicitantes de la TGS por discapacidad. Esto sucede fundamentalmente por dos motivos, un primer motivo se relaciona con que se trata de una participación voluntaria dado que no forma parte del procedimiento del trámite, así como no interfiere en la adjudicación de la prestación; un segundo motivo es que los participantes son derivados por los funcionarios cuando las personas están realizando el trámite y no en todos los casos se les informa que pueden acceder al estudio.

Los criterios de inclusión en el estudio fueron ser mayor de 14 años y presentar alguna limitación funcional o diagnóstico médico que demuestre la presencia de una o más deficiencias.

\section{Instrumentos}

El relevamiento de información se hizo a partir de la aplicación de dos instrumentos. El primero, en orden de aplicación, fue un cuestionario sociodemográfico compuesto por variables relacionadas con la composición del núcleo familiar, ingresos económicos, condiciones de salud, acceso a los servicios de salud, trayectoria educativa y laboral, así como necesidad de ayuda de terceras personas para determinar el perfil epidemiológico. Cada una de las variables cuenta con preguntas cerradas, haciendo un total de 40. En segundo lugar con el Cuestionario para la Evaluación de discapacidad de la OMS (versión española, OMS, 2009) se determinará la situación de salud mental, la severidad de la discapacidad, su naturaleza y su grado de los usuarios de la tarjeta, diferenciando discapacidad intelectual (DI) de las problemáticas de salud por diagnóstico psicopatológico. El WHO-DAS II (OMS, 2009) recoge información socio-demográfica (sexo, edad, estado civil, años en centro educativo, tipo de inserción laboral) y consta de 36 ítems divididos en 6 dominios (comprensión y comunicación; capacidad para moverse en el entorno; cuidado personal; relacionamiento con otras personas; actividades de la vida diaria; participación en la sociedad). Cada dominio se compone de preguntas cuyas respuestas corresponden a una escala Likert, con una 
puntuación de 1 a 5, representando la severidad de la limitación, siendo las opciones: ninguna, leve, moderada, severa y extrema. El instrumento permite una evaluación tanto por dominio como a nivel global, sobre la severidad de la discapacidad. En necesario resaltar que dentro de la consigna de aplicación, el instrumento pide a los entrevistados que sus repuestas sean pensando en los últimos 30 días.

\section{Procedimiento}

La participación, voluntaria, en la entrevista implica la aplicación de dos instrumentos, requiriendo una duración de entre 30 y 60 minutos. Se solicita consentimiento informado a cada participante en que se garantizan los resguardos éticos y los criterios de confidencialidad de los datos.

Luego de cada entrevista se elabora un informe que contiene la valoración del grado de discapacidad, con aspectos biográficos relevantes y del contexto actual de la persona. Estos informes quedan a disposición de las personas participantes del estudio, en resguardo del Archivo de Historias Clínicas de la Facultad de Psicología.

\section{Resultados}

Con relación a los resultados en función del primer objetivo, acerca de identificar el perfil de la población y el grado de discapacidad, se obtiene la siguiente información.

De las personas entrevistadas 50,6\% fueron hombres, de acuerdo con la edad, ordenados por frecuencia, se distribuyeron de la siguiente manera: 78,9\% de los que fueron entrevistados tienen entre 20 y 59 años, el 16,5\% son personas entre 60 y 85 años; el restante 4,5\% tienen entre 15 y 19 años. Otras características se presentan a lo largo de los resultados en función de los objetivos específicos propuestos, tales como nivel educativo o tipo de actividad.

El tipo de discapacidad de los entrevistados fueron en su mayoría de origen mental en un $42,9 \%$, siguiendo por origen intelectual con un $26,7 \%$, origen físico con un $14,2 \%$ y los que declaran tener más de una discapacidad o limitación con 10,5\%. Con menos del $5 \%$ están las discapacidades de origen visceral $(4,5 \%)$ y las sensitivas $(0,8 \%)$.

Según los resultados arrojados por el WHO DAS 2.0 (OMS, 2009), la mayoría de la población valorada presenta una discapacidad moderada (37,2\%), le sigue discapacidad ligera $(35,6 \%)$ y un menor porcentaje discapacidad grave $(24,3 \%)$. No hay situaciones de discapacidad completas. Se encontró un pequeño porcentaje de personas que no arrojaban resultados de discapacidad (2,8\%).

En relación con el origen de la discapacidad, el 85,0\% declara que es a partir de una patología. El resto de los casos se distribuyen con porcentajes menores al $4,0 \%$ : problemas de parto $(3,2 \%)$, accidentes de tránsito $(2,8 \%)$, congénito $(2,4 \%)$, accidentes/condiciones laborales (2,0\%), accidentes domésticos/deportivos $(0,8 \%)$, amputación $(0,4 \%)$, intoxicación química $(0,4 \%)$ y mala praxis $(0,4 \%)$. Es importante resaltar, que la información consignada refiere a lo que la persona conoce de su 
historia, con relación al origen de la discapacidad esta información puede ser por conocimiento directo, cuando es un comienzo en la edad joven o adulta, porque tuvo acceso a su historia clínica o por el relato de otra persona. Un ejemplo de esto es cuando se elige la respuesta "problemas de parto", esta opción responde a una experiencia vital no clarificada sobre su alcance, puede deberse a un problema congénito, a complicaciones de salud al momento del parto o una mala praxis. Esta información, tan importante como la de los informes técnicos, se basa en la opinión de la persona acerca de la percepción que tiene sobre su propia discapacidad.

Por otra parte, de los entrevistados el 83,8\% declara conocer su diagnóstico y sólo el $7,3 \%$ no presenta un diagnóstico claro al momento de la entrevista; los diagnósticos que presentan las personas valoradas se describen en la Tabla 1.

Tal como se desprende de la Tabla 1 el mayor porcentaje de las personas entrevistadas tienen un diagnóstico vinculado a problemas de salud mental, le sigue discapacidad intelectual y luego las vinculadas al sistema osteo-articular. Con una mayor dispersión aparecen otros diagnósticos a los que se le adjudica la razón de presencia de la discapacidad.

Tabla 1. Diagnósticos de las personas entrevistadas

\begin{tabular}{lrr}
\hline \multicolumn{1}{c}{ Diag. $\begin{array}{c}\text { * } \\
\text { Trastorno psiquiátrico definido }\end{array}$} & $\mathbf{F}^{*}$ & 46,6 \\
Discapacidad intelectual & 50 & 20,2 \\
Patologías específicas vinculadas al sistema óseo & 27 & 10,9 \\
No definido & 18 & 7,3 \\
Trastornos neuromotrices & 9 & 3,6 \\
Síndrome de Down & 8 & 3,2 \\
Accidente Cerebro Vascular & 4 & 1,2 \\
Insuficiencia renal crónica & 3 & 1,2 \\
Amputación & 2 & 0,8 \\
Epilepsia & 2 & 0,8 \\
EPOC & 2 & 0,8 \\
Sordera/Hipoacusia & 2 & 0,8 \\
Cardiopatía & 1 & 0,4 \\
HIV & 1 & 0,4 \\
Oncológico & 1 & 0,4 \\
Patología inmunológica & 1 & 0,4 \\
Síndrome de Charcot & 1 & 0,4 \\
Total & 247 & 100 \\
\hline
\end{tabular}

Nota: $(\ddagger D)$ : diagnóstico, $\left({ }^{\star} \mathrm{F}\right)$ : frecuencia, $(\dagger \mathrm{P})$ : porcentaje.

Las categorías utilizadas en la Tabla 1 , se generaron luego de unificar en categorías la diversidad de diagnósticos y la forma de ser registrados para así poder identificar las mismas patologías. Un ejemplo de esto es que para poder llegar al valor de personas entrevistadas con Discapacidad intelectual, se tuvo que agrupar a los que figuraban como "retardo mental", "retraso mental" y "déficit intelectual". Estos diferentes usos 
terminológicos dan cuenta de la diversidad de denominaciones utilizadas y por tanto la falta de actualización en el uso de terminología que refleja las concepciones de los técnicos que ofrecen el diagnóstico.

Como se mencionó anteriormente, si las personas que están gestionando la TGS, ya sea renovación o por primera vez, perciben la prestación de transferencia económica del BPS, no necesita información médica para la gestión. Para estos casos, se les pregunta a los entrevistados si conocían o tenían un diagnóstico. Es decir, que el porcentaje de diagnósticos "No definidos" responden no sólo a no tener información médica sino que además la persona con discapacidad y/o su familiar no tienen conocimiento de la patología.

Con relación al segundo objetivo planteado, se observa que el uso fundamental de la TGS es el beneficio de transporte con boleto gratuito. Se puede observar el predominio en el uso de transporte para asistir a tratamientos, mantener su tratamiento farmacológico y control de salud. El 85,2\% de los entrevistados han asistido a control de salud en los últimos 6 meses; un alto porcentaje mantiene los controles mensuales con los médicos tratantes, ya sean generales o especialistas. El $43,5 \%$ declara que la última consulta con su médico fue para control general, el 42,3\% fue para renovación o cambio de la medicación. Solo un 10,9\% utilizó la consulta para plantear una problemática nueva. El resto de los entrevistados se distribuyen en un 2,0\% no recuerda el motivo de la consulta y el 0,9 fue previo a una internación. Un $87,8 \%$ tienen tratamiento farmacológico; de los cuales el $4,3 \%$ no recuerda ni el nombre ni para qué los toma.

Con respecto a servicios de Rehabilitación, declaran encontrarse en rehabilitación el 59,9\% de los entrevistados. El 19,0\% manifiesta no estar actualmente en rehabilitación pero sí hizo en algún momento y el 21,1\% dice no haber hecho rehabilitación nunca.

A estos dos últimos grupos -no realizan actualmente y nunca realizaron-se les preguntó cuáles eran las razones que motivaron a esta negativa con respecto a la rehabilitación. Las respuestas con mayor frecuencia fueron que "No sabían" la razón $(20,9 \%)$ y haber terminado con el proceso (20,0\%). El 30,0\% está asociado a restricciones para poder acceder a los espacios de rehabilitación ya sea por falta de accesibilidad, falta de información sobre dónde puede haber el servicio, que no se le haya indicado por parte de un equipo técnico, destrato del personal, dificultades económicas, inexistencia del servicio, entre otras. Un $26,0 \%$ se asocian a toma de decisiones por parte de los entrevistados, en las que puede estar condicionando la falta de información y algunos aspectos propios del tipo de discapacidad que presenta; como el caso de discapacidades mentales donde puede existir desgano o falta de interés. Hay un 4,8\% que manifiestan que tuvieron que dejar de asistir debido a problemas asociados a su condición de salud como empujes, intervenciones quirúrgicas, recaídas, entre otras.

Por otro lado, de quienes sí manifiestan estar realizando algún proceso de rehabilitación, el $88,3 \%$ si bien pueden estar realizando más de una a la vez, se centran en el sector de la salud asociados a las funciones corporales y generalmente 
desde una mirada biomédica, más que integral.

Una de las preguntas que surge es qué entienden las personas con discapacidad y sus familiares por rehabilitación y si eso puede estar sesgando las respuestas y las decisiones en relación con la realización o no de las terapias e intervenciones indicadas por los técnicos especialistas.

Según Molina-Achury et al. (2016) se entiende la rehabilitación integral como un proceso que intenta dar respuesta a las necesidades vinculadas a las capacidades y funcionamientos de los sujetos y colectivos. Que se basa en la articulación de políticas, programas, servicios e iniciativas del Estado y la sociedad. Lo hace desde una perspectiva de derechos que fomenta la autonomía y la libertad de los sujetos, la intersectorial de las acciones y las distintas formas de organización social.

Teniendo en cuenta esa definición, la rehabilitación en discapacidad es, la mayoría de las veces, abordada como si fuera similar a enfermedades agudas, donde se aplican "por un tiempo" algunas acciones aisladas, generalmente centradas en los aspectos biomédicos. O programas específicos y finitos en el tiempo de trabajo o educación, de los que se espera que, una vez que finalicen, hayan solucionado todas las dificultades que las personas con discapacidad y sus familias puedan presentar (Ley de Protección Integral de Personas con Discapacidad [LPIPD], 2010)

Una de las consecuencias más directa de este abordaje médico de la rehabilitación para personas con discapacidad es que no se aprovecha al máximo el potencial que tiene vinculado a la mejora del desempeño funcional, la prevención del agravamiento de la severidad y la promoción de la autonomía (OMS, 2011). Si a esto se le suman las relaciones de mercado que pueden aparecer vinculadas al acceso y oferta de rehabilitación, se transforma, para aquellos que no puedan acceder a la misma, en un círculo de exclusión. El enfoque de derechos aparece para reposicionar la rehabilitación como posibilidad de inclusión social y participación (Ley de Protección Integral de Personas con Discapacidad [LPIPD], 2010).

En definitiva, cuando se consulta sobre las actividades, el servicio de la TGS se usa en mayor medida para el control y los cuidados de la salud $(36,2 \%)$ y la realización de trámites (16,6\%). Alrededor del $14,0 \%$ se nuclean las actividades vinculadas a la visita a familiares y amigos y el ocio. Las actividades para las que menos se utiliza son las relacionadas la educación (10,3\%), el trabajo $(6,6 \%)$ y los cuidados $(1,7 \%)$.

Los datos vinculados al uso de la prestación son coherentes con la alta adhesión a las consultas con médicos, la gestión de la medicación y demás aspectos vinculados a la condición de salud o también llamados biomédicos y la poca inserción educativa y laboral. Se puede pensar que analizar el uso del transporte da una dimensión de los niveles de participación social de los usuarios, lo que requeriría también profundizar en el tema. Muchas veces se condiciona la falta de participación de las personas en situación de discapacidad por dificultades de acceso al transporte, sin embargo cabría preguntarse dónde están puestas las barreras de participación cuando acceso al transporte no constituye un problema. 


\begin{tabular}{lcr}
\hline \multicolumn{2}{c}{ Tabla 2. Actividades en las que los entrevistados declaran necesitar ayuda } \\
\hline \multicolumn{1}{c}{ Act. Ayuda (\$) } & $\mathbf{F}^{*}$ & $\mathbf{P} \dagger$ \\
\hline Para limpiar & 49 & $23,0 \%$ \\
Para cocinar & 39 & $18,3 \%$ \\
Para poder desplazarse fuera del hogar & 39 & $18,3 \%$ \\
Para participar en actividades socioculturales & 31 & $14,6 \%$ \\
Para aseo personal & 25 & $11,7 \%$ \\
Para estudiar & 12 & $5,6 \%$ \\
Para la alimentación & 9 & $4,2 \%$ \\
Para trabajar & 6 & $2,8 \%$ \\
Para poder desplazarse dentro del hogar & 3 & $1,4 \%$ \\
\hline
\end{tabular}

Nota: $(\ddagger)$ : actividades en las que necesita ayuda, $\left({ }^{\star} \mathrm{F}\right)$ : frecuencia, $(\dagger \mathrm{P})$ : porcentaje.

El tercer objetivo, se propone explorar la necesidad de apoyo de una tercera persona para realizar las actividades de la vida diaria.

La necesidad de apoyo fue explorada mediante la pregunta sobre la necesidad de ayuda percibida. De ella surge que un $66,4 \%$ declara no necesitar ayuda en los quehaceres de la vida diaria; de estos el $14,0 \%$ presentan una discapacidad grave.

A los que sí declararon necesitar ayuda $(33,6 \%)$, se les consultó en qué tipos de actividades precisan asistencia. En la Tabla 2 se presentan los resultados. Es necesario tener en cuenta que los entrevistadas podían elegir más de una respuesta, por lo que los porcentajes sobre la cantidad de veces que se seleccionó esa categoría y no sobre el total de los entrevistados.

De los resultados planteados, estudiar y trabajar no aparecen dentro de las actividades identificadas con mayor necesidad de ayuda. Esto puede deberse a dos aspectos relacionados entre sí; en primer lugar, a la población entrevistada tiene poca inclusión laboral y educativa, lo que lleva al segundo aspecto, a que la población entrevistada no identifica el trabajo o la educación como una actividad a realizar y por lo tanto no puede dimensionar si precisa ayuda o no.

Las tareas más vinculadas a los quehaceres del hogar, como limpiar y cocinar, son las actividades con mayor frecuencia de respuesta y esto puede deberse a que son actividades necesarias para la cotidianeidad por lo que es fácil de identificar como una necesidad y por otro lado, son tareas que requieren de planificación además de acciones de ejecución por lo que para diversos tipos de discapacidad pueden ser actividades que generen dificultad.

Con respecto al cuarto objetivo, los resultados para determinar necesidades de acceso a servicios e inclusión social, se exploraron con la información vinculada las actividades de participación social, excluyendo aquí los servicios de salud que fueron explorados en los resultados obtenidos para el segundo objetivo.

Es así que un dato importante para la inclusión social, se vincula con las posibilidades de acceso a la educación. En relación con la trayectoria educativa el 88,7\% relata haber 
asistido "alguna vez", dentro de este grupo los porcentajes más altos en relación al nivel educativo más alto alcanzado son los que tienen primaria completa $(24,2 \%)$, primaria incompleta $(16,9 \%)$ y educación especial $(13,7 \%)$. De los entrevistados un $8,1 \%$ manifiesta estar asistiendo a algún espacio de educación formal y un 3,2\% dice no haber asistido nunca.

Dado que se trata de población mayor a 18 años, otro de los ámbitos explorados se vincula con la actividad laboral y/o si recibe algún tipo de ingresos. Si tomamos en cuenta los principales ingresos, las personas entrevistadas se definen en su mayoría $(40,5 \%)$ como pensionista por invalidez o jubilado por discapacidad - ambas prestaciones económicas de la seguridad social. De ellos, un 37,0\% presentan una discapacidad moderada, un 32,9\% una discapacidad ligera, 27,4\% una discapacidad grave y el 2,7\% no presentan discapacidad, según el WHODAS 2.0 (OMS, 2009). Hay un $29,1 \%$ que declaran ser desempleados; de estos el $86,1 \%$ manifiesta que no tiene trabajo debido a su condición de salud. El 12,1\% tiene una jubilación no vinculada a la condición de salud, sino porque cumplió con los años de trabajo y la edad exigida. Sólo el 7,3\% percibe un salario por su trabajo y un 6,5\% está estudiando. En menor número un $2,8 \%$ son cuentapropistas, $1,2 \%$ son responsables de los quehaceres del hogar y un $0,4 \%$ realizan trabajo no remunerado.

Los resultados permiten observar que la mayoría de las personas entrevistadas acceden a los servicios de salud (rehabilitación, tratamiento y control) pero no tanto a espacios educativos, laborales y de ocio. Las personas que solicitan la TGS tienen mayores limitaciones en lo que respecta a las interacciones con otros y la participación y no tanto en limitaciones en las actividades de la vida diaria que involucren actividades puntuales y que se puedan desempeñar de manera individual como el autocuidado y los quehaceres del hogar. Se suma a esto que el 73,3\% de los valorados no realizan actividades laborales o de estudio, aun siendo la mayoría personas que tienen entre 20 y 59 años $(78,9 \%)$.

Para este objetivo, se toma además la dimensión de "relacionamiento con otras personas" que se explora a través del WHO-DAS 2.0 (OMS, 2009), ya que analiza la capacidad de hacer y mantener amigos, de tener relaciones sexuales, de relacionamiento con personas que ya conoce, entre otras. Como en ninguna otra dimensión, la distribución de los resultados sobre la limitación que pueden tener las personas valoradas, es sumamente pareja. Si bien la mayor parte de la población con la que se trabajó contestó no tener limitaciones (27,9\%), un 27,5\% manifiesta tener una limitación grave para este tipo de actividades. Más cerca del 20,0\% se encuentran quienes tienen una limitación ligera $(20,2 \%)$ y una limitación moderada $(22,3 \%)$.

Por otra parte, el 47,4\% declara tener una limitación grave y completa en la dimensión participación de actividades a nivel comunitario, que incluyen recibir un trato con respeto y dignidad, de que su condición de salud no haya afectado sus vínculos familiares y su realidad económica. 


\section{Discusión}

La intendencia de Montevideo tiene una larga trayectoria en la prestación que actualmente se denomina Tarjeta de Gestión Social. Por lo que entiende necesario contar con un instrumento que pueda valorar la discapacidad de los posibles beneficiarios, bajo los mismos parámetros y criterios, tal como lo exigen los paradigmas actuales. Tal como se ha planteado, el criterio para acceder a la prestación se basa en informes médicos y/o técnicos de diversa índole, así como si la persona tiene una prestación por discapacidad de otro organismo público y a la vez pasa por una revisión de valoración médica. El presente estudio muestra que el WHO-DAS II (OMS, 2009) es un instrumento útil a la hora de evaluar la existencia o no de discapacidad, así como su grado, desde una perspectiva integral, que incluye además de la condición de salud, el contexto de la persona. En este sentido, resulta de utilidad para obtener información que permite identificar un perfil poblacional beneficiaria de las prestaciones de la política pública. Asimismo, junto con la aplicación del cuestionario socio-demográfico, se puede obtener información relacionada con la salud, las necesidades de apoyo y la participación social de las personas, lo que redunda en contar con datos para la evaluación y ajuste de las políticas. Además se plantea como valor agregado, el bajo costo de inversión que supone su implementación, con baja exigencia tecnológica, realizable en una entrevista que puede llevar entre 30 y 60 minutos.

A la luz de las concepciones actuales sobre los derechos de las personas con discapacidad, el diseño de las políticas debería basarse en información acerca de sus necesidades de forma tal que, a partir de la presencia de una deficiencia, física o mental, se eliminen las barreras que impidan la participación de las personas en los diferentes ámbitos de la vida y puedan reconocerse sus capacidades, fundamentalmente para tomar sus propias opciones.

Considerando los objetivos planteados, las actividades que realizan las personas que solicitan la TGS están vinculadas a lo que requiere el control y el mantenimiento de su salud y la realización de trámites; y lo que resalta junto a esto es que tienen mayores limitaciones en lo que respecta a las interacciones con otros y la participación y no tanto en limitaciones en las actividades de la vida diaria que involucren tareas puntuales y que se puedan desempeñar de manera individual. Además, la mayoría de los valorados no realizan actividades laborales o de estudio. La prestación otorgada por la IM puede funcionar, entonces, de apoyo para la promoción de autonomía, ya que los entrevistados declararon que la mayoría de las actividades de la vida diaria para las que requieren del uso de transporte se realizan de manera independiente. Como plantean Badía Corbella y Longo Araújo de Melo (2009) la posibilidad de elección que brindan algunas prácticas, como el transitar de forma autónoma por la ciudad, se constituyen en herramientas para la toma de decisiones y la participación en la comunidad.

Como surge de los resultados, es claro que existe una diversidad de experiencias y trayectorias de las personas que solicitan la TGS. La información recabada, permite 
dar cuenta que la discapacidad es considerada a partir de diferentes diagnósticos, de diferentes criterios, dependiendo del organismo que la determine. También ello determina la adjudicación de la TGS en forma automática cuando las personas tienen ya una evaluación técnica realizada por otro organismo, por ejemplo, la pensión por invalidez. Se puede observar que en estos casos, el instrumento utilizado ha permitido discriminar diferentes grados de discapacidad, aun cuando la pensión por invalidez se otorga únicamente en casos de discapacidad severa.

En relación a la salud, las personas cuentan con cobertura y acceso, llegando incluso a ser la actividad principal de las personas (controles farmacológicos, sanitarios generales o especialistas) Sin embargo dicha cobertura no da cuenta de una trayectoria vinculada a la atención integral de la discapacidad, sino a tratamientos de diagnósticos específicos. Esto se observa también frente a la consulta sobre rehabilitación. La mayoría de los entrevistado acceden a los servicios y las prestaciones de salud (rehabilitación, tratamiento y control), pero existen vacíos en relación con los diagnósticos, a qué se entiende por rehabilitación y la consideración de que la consulta mensual y la ingesta adecuada de la medicación es el abordaje sanitario que las personas con discapacidad precisan para su inclusión y mejora de la calidad de vida (França et al., 2010). También es significativo que no todos cuenten con informes técnicos o no sean accesibles para quien debe tomar la determinación de otorgar una prestación.

Aunque haya adhesión alta al tratamiento farmacológico y constancia en la visita médica no todas las personas están informadas de su diagnóstico; tienen dificultad en identificar cuál es el origen de la discapacidad (ejemplo de problema de parto y cómo puede quedar oculto el dato por no saber sobre la historia en relación a su situación de discapacidad). La rehabilitación es casi confundida con las consultas médicas y se basan en la rehabilitación funcional y no integral. Sobre los que no han hecho o no actualmente, priman las razones de desconocimiento sobre lo que es la rehabilitación o las dificultades de acceder a la misma. Como plantea Puga (2005) si lo que priman son el tratamiento de lo clínico de manera no integral y las limitaciones que puede presentar una persona con discapacidad son vistas como patologías, se termina responsabilizando a la persona que consulta y anula la posibilidad de ver a la discapacidad como una responsabilidad colectiva.

Si bien este estudio presenta limitaciones en cuanto al tamaño de la muestra y por ende la representatividad de los resultados, permite avanzar en la necesidad de establecer criterios técnicos e instrumentos unificados para el establecimiento de diagnósticos, la valoración de la discapacidad, la rehabilitación y el tratamiento. Los resultados obtenidos permiten advertir de la visión parcial con la que se abordan las situaciones de discapacidad, en general amparadas o en un diagnóstico médico, que no toma en cuenta barreras y facilitadores para su inclusión social.

Otra limitación del estudio refiere a que se trata de una población sesgada en primer lugar al departamento de Montevideo y en segundo lugar a aquellos que acceden a solicitar la TGS, además al ser de participación voluntaria, reduce el número de 
participantes frente a los que podrían incluirse si fuera parte del procedimiento de otorgamiento de la prestación.

Creemos necesario avanzar en esta línea de estudio que permita profundizar en las diferentes dimensiones que determinan el perfil epidemiológico; así como consideramos que sería de utilidad realizar un estudio sobre los criterios e instrumentos que utilizan los profesionales de la salud para aportar informes técnicos sobre la situación de discapacidad, más allá de los baremos o criterios que utilizan las instituciones para otorgar certificados y prestaciones.

\section{Referencias}

Alarcón, J. (2009). Epidemiología: concepto, usos y perspectivas. Revista Peruana de Epidemiología, 13(1), 1-3.

Ayuso-Mateos, J. L., Nieto-Moreno, M., SánchezMoreno, J. y Vázquez-Barquero, J. L. (2006). Clasificación Internacional del Funcionamiento, la Discapacidad y la Salud (CIF): Aplicabilidad y utilidad en la práctica clínica. Medicina Clínica, 126(12), 461-466. HTTPS://DOI. ORG/10.1157/13086326.

Badía Corbella, M. y Longo Araújo de Melo, E. (2009). El ocio en las personas con discapacidad intelectual: Participación y calidad de vida a través de las actividades de ocio. Siglo Cero Revista Española sobre Discapacidad Intelectual, 40(3), 30-44

Ballerini, A, Figueras, L. y Del Carlo, C. (2020). ¿Es la salud un derecho? Políticas públicas en tiempos de neoliberalismo. Revista de Psicología, 19(1), 3247. HTTPS://DX.DOI.ORG/10.24215/2422572XEO48

Cobas Ruíz, M. C., Peña, E. Z., Calatayud, F. M., Pereira, E. I., Hernández, A. J. y Sosa, M. V. (2010). Caracterización epidemiológica de las personas con discapacidad en Cuba. Revista Cubana de Salud Pública, 36(4), 306-316.

França, I. S. X. de, Pagliuca, L. M. F., Baptista, R S., França, E. G. de, Coura, A. S. y Souza, J. A. de. (2010). Violência simbólica no acesso das pessoas com deficiência às unidades básicas de saúde. Revista Brasileira de Enfermagem 63(6), 964-970. HTTPS://DOI.ORG/10.1590/soo34 71672010000600015
Instituto Nacional de Estadísticas (2005, abril) Primer estudio nacional de la discapacidad en Chile. Fondo Nacional de Discapacidad. HTtPS://WWW.SENADIS.GOB.CL/PAG/136/1196/ RESULTADOS_ENDISC_I

Jiménez Lara, A. (2010). Salud pública y discapacidad Planteamientos y propuestas del sector social de la discapacidad articulado en torno al CERMI ante la futura Ley estatal de Salud Pública (1era ed.). Grupo Editorial Cinca, S. A.

Ley de Protección Integral de Personas con Discapacidad $n^{\circ} 18651$ República Oriental del Uruguay (2010). HTTPs://Www.IMPo.com.uY/ BASES/LEYES/18651-2010.

Molina-Achury, N., Mogollón-Pérez, A., BalantaCobo, P., Moreno-Angarita, M., HernándezJaramillo, B. y Rojas-Castillo, C. (2016). Lógicas dominantes en la formulación de políticas y organización de servicios que inciden en el acceso a la rehabilitación integral. Revista Gerencia y Politicas de Salud, 15(30), 94-106.

Núñez, I. (2011, Mayo). Personas con discapacidad en Uruguay: algunas cifras del censo 2011. Banco de Previsión Social. HTtPs://www.BPS. GUB.UY/BPS/FILE/8195/1/44._PERSONAS_CON_ DISCAPACIDAD_EN_URUGUAY._ALGUNAS CIFRAS_DEL_CENSO_2011._NUNEZ.PDF

Organización Mundial de la Salud (2001, Setiembre). Clasificación Internacional del funcionamiento, de la Discapacidad y de la Salud. Organización Mundial de la Salud. HTtPs://ASPACE.ORG/ASSETS/ UPLOADS/PUBLICACIONES/E74E4-CIF_2001.PDF 
Organización Mundial de la Salud (2009). Medición de la salud y la discapacidad. Manual para el Cuestionario de Evaluación de la Discapacidad de la OMS. WHODAS 2.0. IMSERSO. H T T P S://APPS.WHO.INT/IRIS/BITSTREAM/ HANDLE/ $10665 / 170500 / 9874573309$ _SPA. PDF?SEQUENCE $=1 \&$ ISALLOWED $=Y$

Organización Mundial de la Salud (2011). Informe mundial sobre la discapacidad. Organización Mundial de la Salud. HTTPs://www.who.InT/ DISABILITIES/WORLD_REPORT/2011/ES/

Ortiz Segarra, J. I. (2013). La discapacidad en el Ecuador en cifras, año 2010. Revista de la Facultad de Ciencias Médicas, 31(1), 74-81.

Puga, D. (2005). La dependencia de las personas con discapacidad: Entre lo sanitario y lo social, entre lo privado y lo público. Revista Española de Salud Pública, 79(3), 327-330.
Rodriguez Da Silva, G. (2006). La formación de personal en función de los usos y perspectivas de la Epidemiología. Revista Brasileira de Epidemiologia, 9(1), 24-31. HTTPS://DOI ORG/10.1590/s1415-790X2006000100004

Tamayo, M., Besoaín, Á. y Rebolledo, J. (2018). Determinantes sociales de la salud y discapacidad: Actualizando el modelo de determinación. Gaceta Sanitaria, 32(1), 96-100. нтTPs://DOI. ORG/10.1016/J.GACETA.2016.12.004 